\title{
High frequency jet ventilation during endolaryngeal surgery: a prospective cohort
}

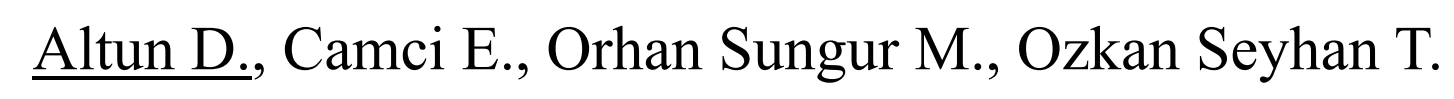

Istanbul University, Istanbul Faculty of Medicine, Department of Anesthesiology and Perioperative Medicine, Turkey

\section{Background and Goal of Study:}

In this prospective observational study, we aimed to identify factors associated with intraoperative complications during endolaryngeal surgery (ELS) with infraglottic high frequency jet ventilation (HFJV) under standardized anesthesia regimen and we described our clinical experience with jet ventilation during ELS in 209 patients.

\section{Materials and methods:}

- 217 patients who underwent ELS with infraglottic HFJV were enrolled.

- Anaesthesia was induced with midazolam, remifentanil and propofol. Mivacurium was used for neuromuscular blockade. Infraglottic jet ventilation catheter was placed with the aid of a laryngoscope and anesthesia was maintained with propofol and remifentanil infusion under bispectral index guidance.

- Demographic (age, gender, comorbidities including respiratory and cardiovascular disease) and operative (type of surgery, operation time, previous laryngeal operations) data were noted. Hemodynamics (heart rate, mean arterial pressure), $\mathrm{SpO}_{2}$ and end-tidal $\mathrm{CO}_{2}$ were monitored and were recorded at regular intervals.

- Complications such as hypoxia, hypercarbia, barotrauma, equipment failure, and requirement for conventional ventilation were also documented.

\section{- Results:}

- 230 patients undergoing minor laryngotracheal surgery were screened and 217 consenting patients were enrolled.

- During anaesthetic induction, eight patients were excluded and 209 patients were included in the statistical analysis. Surgery was completed with initial HFJV settings in 190 patients.

- Additional steps to maintain adequate gas exchange were required in 15 patients, including orotracheal intubation in four of these patients.

- Orotracheal intubation was further needed in four patients due to surgical preference.

- During anaesthesia emergence, laryngeal mask insertion was applied in five patients.
Table 1 Demographic data and surgical indications

\begin{tabular}{|c|c|}
\hline Variable & Data \\
\hline Age (years) & $52.3 \pm 11$ \\
\hline Gender: Male/ Female & $147(70.3 \%) / 62(29.7 \%)$ \\
\hline ASA status $(1 / 2 / 3)$ & $70(33.5 \%) / 119(56.9 \%) / 20(9.6 \%)$ \\
\hline $\begin{array}{l}\text { History of respiratory } \\
\text { disease }\end{array}$ & $62(29.67 \%)$ \\
\hline $\begin{array}{l}\text { History of cardiovascular } \\
\text { disease }\end{array}$ & $66(31.6 \%)$ \\
\hline Mallampati $(1 / 2 / 3 / 4)$ & $\begin{array}{l}41(19.6 \%) / 129(61.7 \%) / 28(13.4 \%) / \\
11(5.3 \%)\end{array}$ \\
\hline $\begin{array}{l}\text { Cormack-Lehane } \\
\text { (I/II/III/IV) }\end{array}$ & $\begin{array}{l}47(22.5 \%) / 131(62.7 \%) / 21(10 \%) / \\
10(4.8 \%)\end{array}$ \\
\hline Body mass index $\left(\mathrm{kg} \cdot \mathrm{m}^{-2}\right)$ & $25.66 \pm 3.35$ \\
\hline $\begin{array}{l}\text { Benign laryngeal } \\
\text { pathologies (nodule, polip, } \\
\text { web, Reinke aedema, } \\
\text { phonosurgery) }\end{array}$ & $131(62.7 \%)$ \\
\hline Vocal cord paralysis & $25(12 \%)$ \\
\hline $\begin{array}{l}\text { Diagnostic biopsy for } \\
\text { vocal cord neoplasia }\end{array}$ & $30(14 \%)$ \\
\hline Subglottic stenosis & $18(8.6 \%)$ \\
\hline Tracheal stenosis & $5(2.4 \%)$ \\
\hline
\end{tabular}

Data are given as mean $\pm \mathrm{SD}$ or number $(\%)$

Table 2 The evaluation of complications

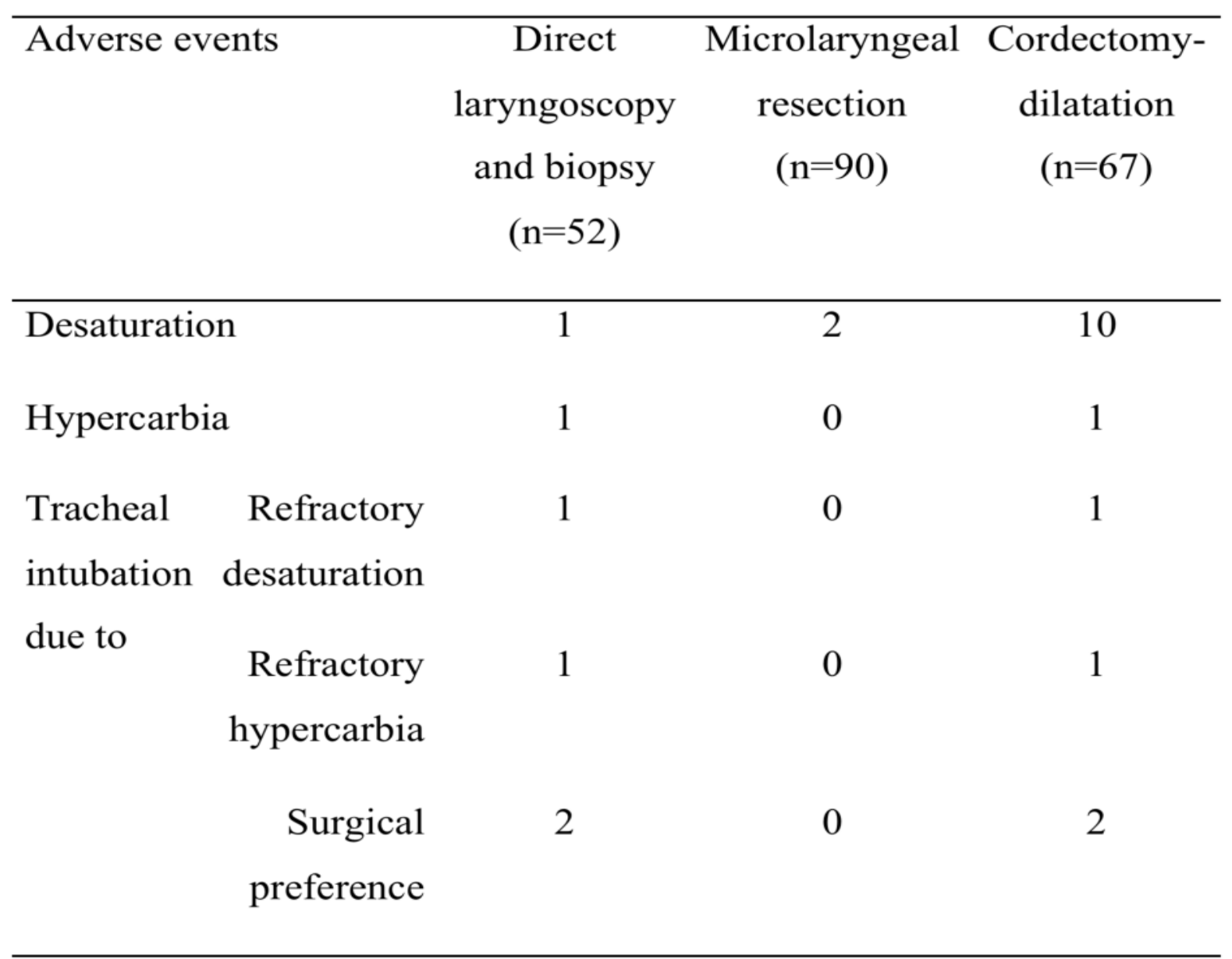

\section{Discussion:}

High frequency jet ventilation via infraglottic catheter is an artificial breathing technique during endolaryngeal interventions that provides adequate oxygenation, an unobstructed view of the surgical field and safe conditions for laser use. ${ }^{1}$

\section{Conclusion(s):}

Jet ventilation with suggested anaesthesia regimen is a good alternative ventilation in endolaryngeal surgery.

Key words: Anesthesia, airway, jet ventilation

\section{References:}

1. Fritzsche K, Osmers A. Anesthetic management in laryngotracheal surgery. High-frequency jet ventilation as strategy for ventilation during general anesthesia. Der Anaesthesist, 2010;59 (11): 1051-1061. 